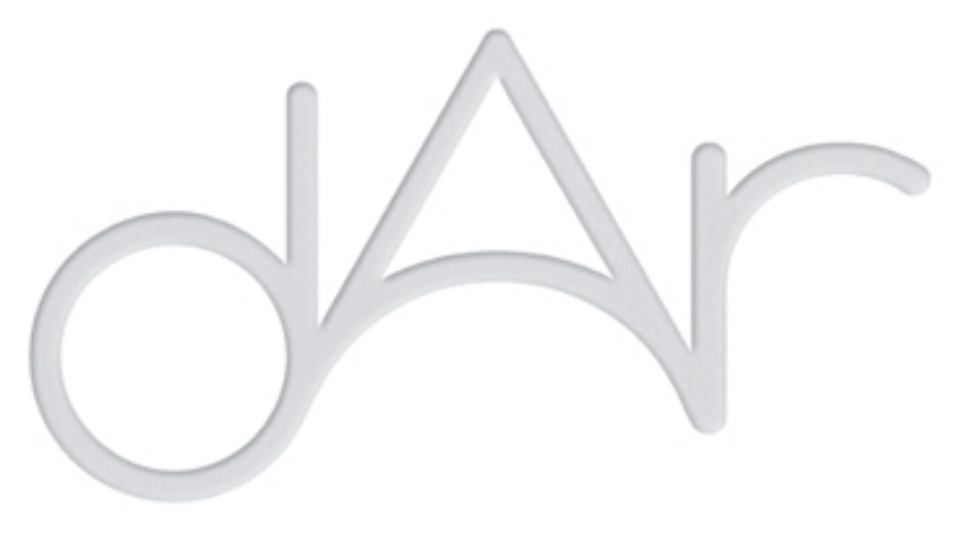

\title{
Valorisation touristique du patrimoine éco-culturel au Maroc méridional: cas des oasis du Draa Moyen
}
Autor(es):
Bentaleb, Aziz; Bouaouinate, Asmae
Publicado por: Imprensa da Universidade de Coimbra
URL
persistente: URI:http://hdl.handle.net/10316.2/44431
DOI: DOI:https://doi.org/10.14195/2182-844X_5_10
Accessed : $\quad$ 26-Apr-2023 03:21:30

A navegação consulta e descarregamento dos títulos inseridos nas Bibliotecas Digitais UC Digitalis, UC Pombalina e UC Impactum, pressupõem a aceitação plena e sem reservas dos Termos e Condições de Uso destas Bibliotecas Digitais, disponíveis em https://digitalis.uc.pt/pt-pt/termos.

Conforme exposto nos referidos Termos e Condições de Uso, o descarregamento de títulos de acesso restrito requer uma licença válida de autorização devendo o utilizador aceder ao(s) documento(s) a partir de um endereço de IP da instituição detentora da supramencionada licença.

Ao utilizador é apenas permitido o descarregamento para uso pessoal, pelo que o emprego do(s) título(s) descarregado(s) para outro fim, designadamente comercial, carece de autorização do respetivo autor ou editor da obra.

Na medida em que todas as obras da UC Digitalis se encontram protegidas pelo Código do Direito de Autor e Direitos Conexos e demais legislação aplicável, toda a cópia, parcial ou total, deste documento, nos casos em que é legalmente admitida, deverá conter ou fazer-se acompanhar por este aviso.

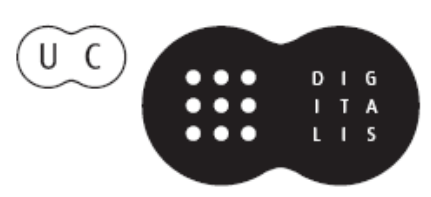



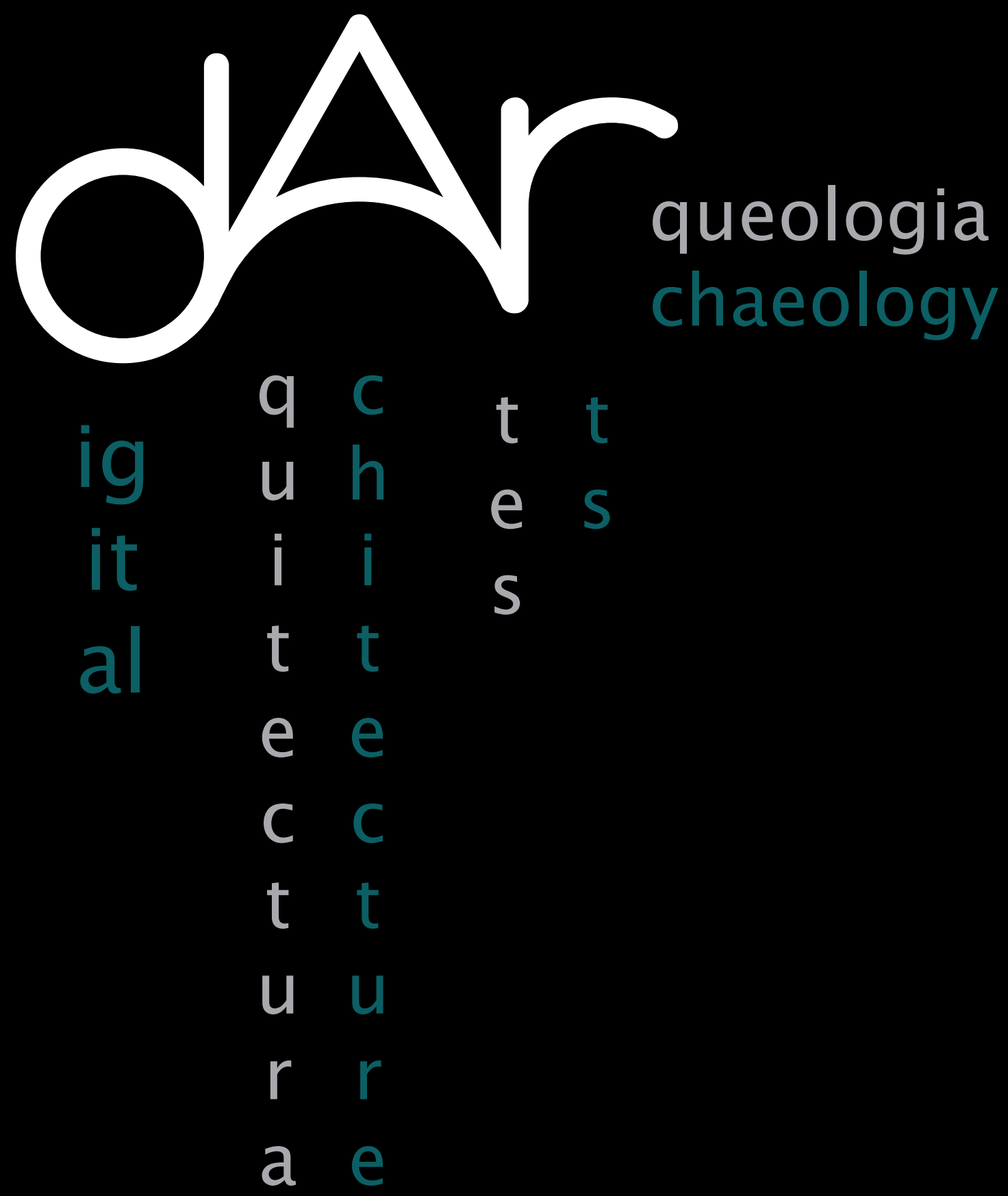

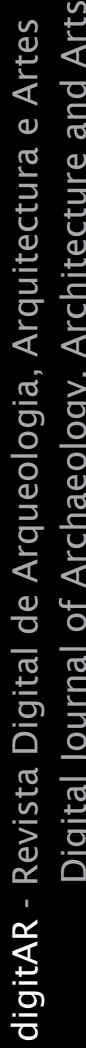




\title{
VAlorisation touristiQue du PATRIMOINe ÉCo-CUlturel aU Maroc méridional, CAS des oAsis du DraA Moyen
}

\author{
Dr. Aziz Bentaleb, CEHE, IRCAM \\ Laboratoire Ladyss, UMR 7533, Université de Paris-Ouest
}

Dr. Asmae Bouaouinate, FLSH, Mohammedia

\section{Introduction}

Le développement du tourisme dans les oasis du Draa à travers la valorisation des produits du terroir patrimoniaux présente un double intérêt, autant au niveau de l'économie nationale que pour le développement régional.

La mise en œuvre d'une stratégie de développement du secteur touristique dans la zone permettrait d'impulser une dynamique locale, basée sur la bonne gouvernance des ressources patrimoniales territoriales. Toutefois, la promotion du tourisme doit s'appuyer sur les paramètres économiques, mais aussi intégrer la dimension socioculturelle et environnementale locale : écosystèmes, ressources naturelles, patrimoine, traditions, etc. Les stratégies d'aménagement du territoire et du développement de l'éco-tourisme doivent en tenir compte pour promouvoir un développement participatif et intégré. De par ses richesses écologiques et humaines, le Draa constitue un espace de reconnaissance internationale dans la réserve de biosphère des palmeraies du sud marocain. En plus de son poids historique et anthropologique, il recèle des ressources considérables conditionnant l'essentiel des activités des zones oasiennes de l'Anti-Atlas oriental.

L'importance du présent travail résidedans la valorisation etla «touristification» des produits de terroir, notamment ceux liés à la biodiversité (palmier dattier) et les productions culturelles matérielles.

Actuellement, il est admis que le souci de préserver et de mettre en valeur les ressources territoriales rejoint souvent celui de maintenir les savoirs et les traditions locaux. Cette conception est d'autant plus vraie que les sociétés humaines ont modelé leur patrimoine en fonction de leurs préférences culturelles et matérielles. Or, sous l'effet conjugué de plusieurs facteurs tels que la croissance rapide de la population, l'éloignement des marchés internationaux et des procédés de développement, nous assistons de plus en plus à une extinction des systèmes de connaissances locales, de la biodiversité et de la diversité culturelle, lesquels constituent trois systèmes interdépendants.

https:/ /doi.org/10.14195/2182-844X_5_10 


\section{Présentation de la zone d'étude}

Située dans le Sud-Est marocain, vers le trentième parallèle, traversée en son milieu par le méridien $6^{\circ} \mathrm{C}$ ouest, la vallée du Drâa Moyen s'allonge sur environ de $230 \mathrm{~km}$, couvrant plus de $23000 \mathrm{~km}^{2}$. Elle est bordée au nord par le Jebel Saghro, à l'est par la remontée nord du Jebel Bani, au sud par la hamada du Drâa et à l'Ouest par le massif de l'Anti-Atlas. Cet espace d'écosystèmes arides fait partie de la réserve de biosphère des palmeraies du sud marocain (Fig. 1).

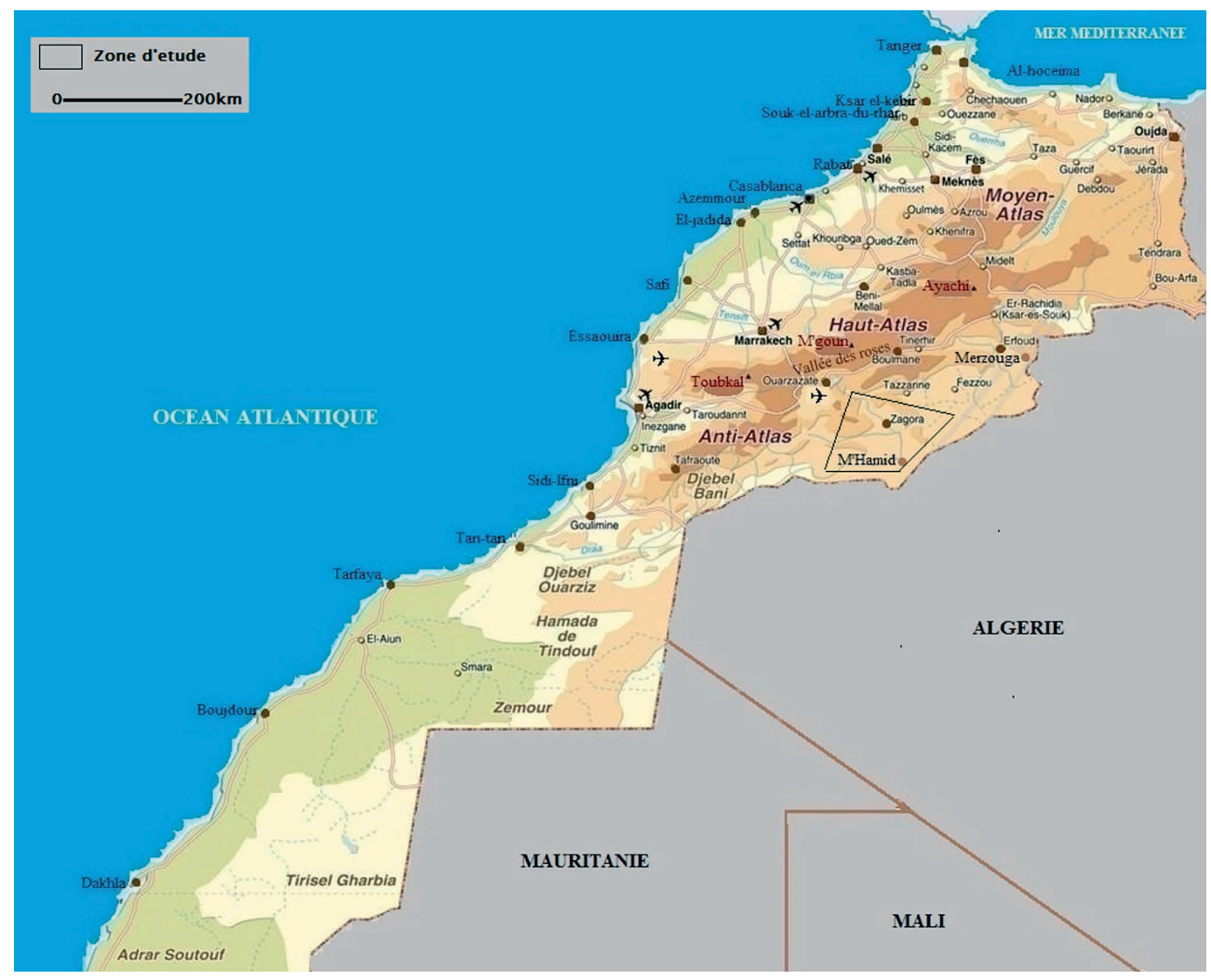

Fig.1 : Situation géographique de la vallée du Draa au Maroc

Le paysage de palmeraies, étirés au milieu d'un vaste espace dénudé, donne l'image d'une richesse non négligeable, la présence d'un oued allogène bordé de terrasses relativement fertiles a sans doute séduit, depuis des temps immémoriaux, différents peuples comme en témoignent les nombreuses 
stations rupestres, d'âges divers, jalonnant la vallée et les régions limitrophes. La position stratégique du Draa lui confère un riche patrimoine génétique phoenicicole (palmier dattier), des ressources floristiques, faunistiques ainsi que des potentialités culturelles très diversifiées (sites historiques, patrimoine immatériel artistique, savoir-faire traditionnels ; gestion participative des eaux d'irrigation, architecture millénaire en pisé).

La valorisation de ces atouts patrimoniaux au service du tourisme représente autant d'éléments, pouvant contribuer à la protection de l'écosystème oasien et, par conséquent, de promouvoir le développement local.

\section{L'agrotourisme, un potentiel générateur de revenus pour les oasiens}

L'agrotourisme est considéré comme l'une des activités exercées à la ferme par les exploitants agricoles ou par des membres de l'exploitation, ayant un certain contenu. C'est-à-dire toutes les activités correspondant à la maîtrise et à l'exploitation d'un cycle biologique, végétal ou animal et constituant une ou plusieurs étapes nécessaires au déroulement de ce cycle ainsi que les activités exercées par un exploitant, qui sont dans le prolongement de l'acte de production ou qui ont pour support l'exploitation. En l'absence de lien avec l'agriculture, il y a obligation d'une immatriculation au registre du commerce (Diser, 1996). Les acteurs de l'agritourisme, composante du tourisme vert, sont des exploitants agricoles qui proposent sur leur exploitation soit une activité de restauration, soit une activité d'hébergement, soit une activité de loisirs et, maintenant, une activité de vente directe de leurs produits. C'est donc l'ensemble des acteurs, qu'ils soient publics, privés ou associatifs, qui proposent des activités d'hébergement, de restauration et de loisirs en espace rural (Jacques et Emmanuelle, 2001).

Le choix de la phoneniciculture comme produit d'agrotourisme dans la zone est justifiée par l'importance de la filière dattière du point de vue stratégique et la place qu'occupe le palmier dattier dans l'économie de la région. La datte représente l'élément moteur de l'intégration de l'exploitation agricole locale à l'économie marchande. Elle constitue, en effet, le principal facteur de monétarisation et continue à générer des revenus importants pour les populations des palmeraies surtout compte tenu de l'image touristique de la région qui fait partie de la réserve de biosphère des oasis du sud marocain (RBOSM), et qui s'est renforcée ces dernières années par la reconnaissance internationale de sa richesse culturelle. 


\subsection{Echelle intrinsèque de la production}

Sur le plan effectif, la région englobe 1.800 .000 pieds. Cependant, le caractère spontané de l'espèce accuse la prédominance des variétés issues de semis ou khalts $(42.2 \%$ de l'effectif productif total). Des efforts sont encore à déployer afin de corriger la densité de peuplement et d'introduire des variétés résistantes au bayoud et de bonne valeur commerciale.

Du point de vue tonnage, la région contribue en moyenne à $50 \%$ de la production nationale. La production des dattes est en moyenne de

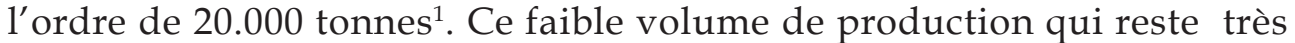
loin des potentialités théoriquement envisageables est caractérisé par son insuffisance et son irrégularité dans le temps et dans l'espace.

L'insuffisance et la grande variabilité de la production sont le résultat d'un environnement défavorable. En dehors des problèmes causés par la maladie du bayoud, le secteur phoénicicole est caractérisé par des pratiques culturales ancestrales de la plantation à la récolte et au conditionnement. D'où l'intérêt de mettre en place une technologie appropriée, en mesure d'accroître la productivité en amont par l'approvisionnement et l'utilisation adéquate des intrants et en aval par la garantie des débouchés.

Dans les périmètres irrigués par le barrage, on signale aussi l'insuffisance de la mobilisation des eaux d'irrigation, laquelle se fait actuellement pour répondre aux besoins des cultures basses (essentiellement les céréales). Il y'a lieu donc d'envisager des travaux de recherche pour améliorer les conditions d'irrigation et la fertilisation du palmier.

\section{2. Acteurs de l'agro-tourisme dans la zone}

L'identification des acteurs qui font des activités agritouristiques a été réalisée au moyen d'une fiche d'enquête auprès des agriculteurs, des opérateurs privés, des associations et de 200 touristes.

Cette enquête (qualitative et quantitative) complétée par des entretiens a touché également des personnes ressources, particulièrement compétentes (techniciens agricoles, agents de développement d'une collectivité locale, délégation de tourisme ...).

1 Subdivision de Zagora (2016) 


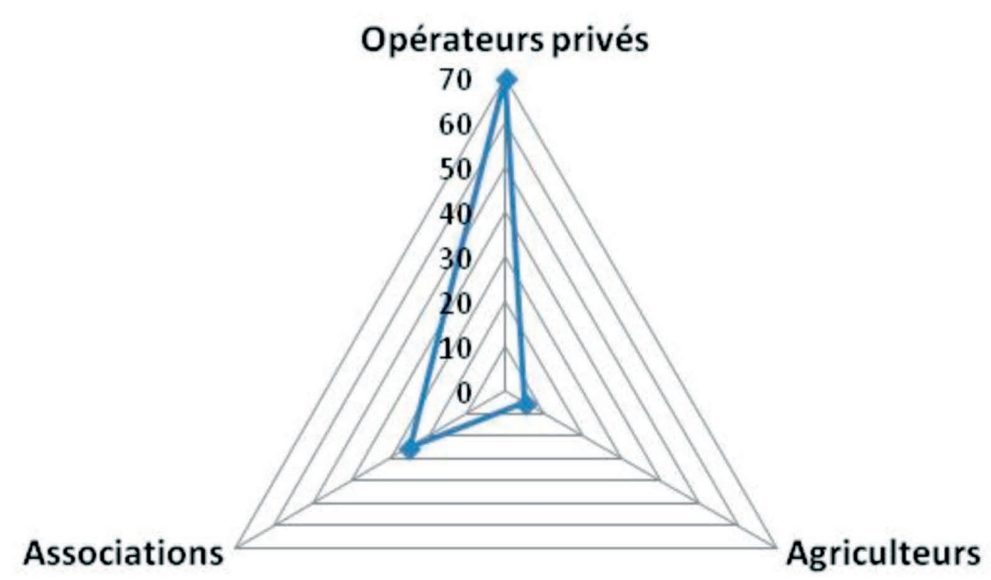

Fig. 2 - Les acteurs locaux de l'agrotourisme dans le Draa (\%). (Source : enquête de terrain, 2017)

La lecture de la figure $\mathrm{N}^{\circ} 2$ met en évidence que le développement de l'activité de l'agrotourisme dans la zone est relativement faible et, par conséquent, $\mathrm{n}^{\prime}$ apparaît pas actuellement comme une solution alternative de développement pour la valorisation des produits de terroir, notamment le palmier dattier, arbre magique de désert. L'agrotourisme n'est pratiqué que par $5 \%$ des agriculteurs, faute de sensibilisation et d'encadrement de I'ORMVAO (Office Régionale de Mise en Valeur Agricole de Ouarzazate) et des services du tourisme à l'échelle locale. L'agritourisme, ou principalement le tourisme oasien (paysages, culture, tradition, etc.) dans la zone est essentiellement le fruit de pratiques individuelles dirigées à distances par des opérateurs privés (agences de voyages) qui représentent $70 \%$ des pratiquants, non seulement d'agrotourisme mais aussi du tourisme culturel et surtout désertique. Les associations locales ne contribuent qu'à hauteur de $25 \%$ dans la valorisation touristique des produits agricoles en raison du manque de concertation, de coordination entre les acteurs du tourisme et de l'absence des politiques spécifiques de développement agrotouristique au niveau de la zone. Effectivement, leurs efforts se focalisent sur la sauvegarde du palmier dattier et la réalisation des actions socioculturelles. 


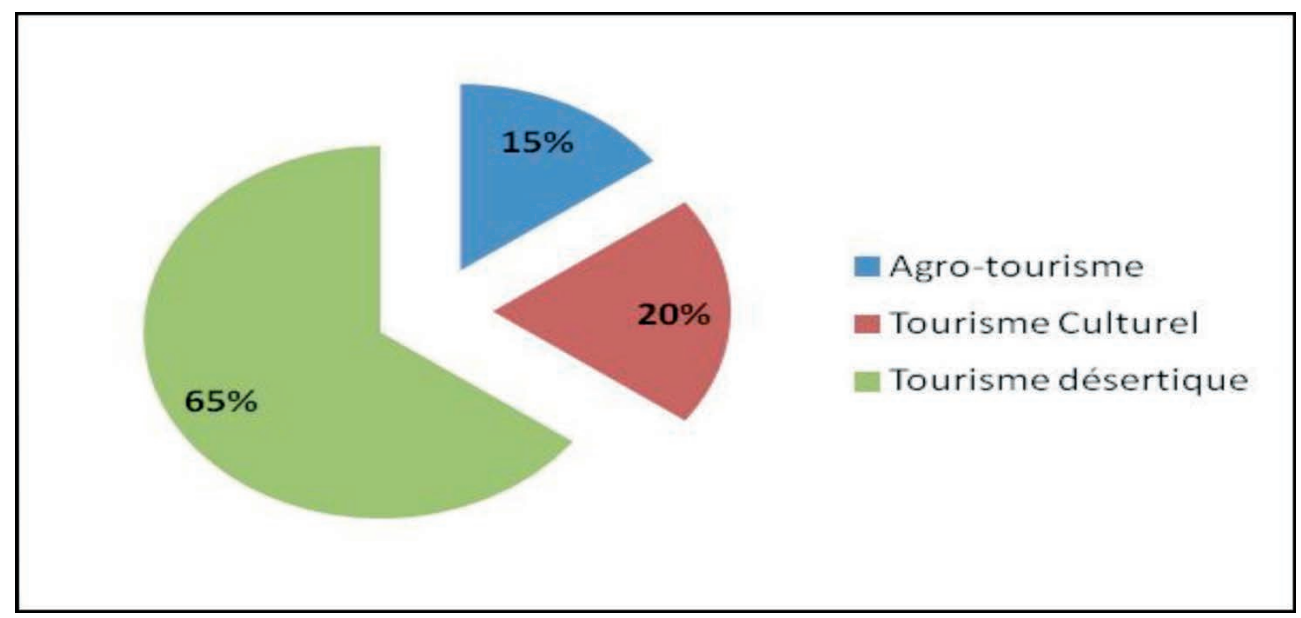

Fig. 3 - Catégorie du tourisme recherché par les touristes dans la zone

En outre, les investigations de terrain montrent que le touriste lui-même, de passage dans la vallée, n'a pas un grand intérêt à l'agrotourisme (fig. 3). Seuls $15 \%$ des touristes s'intéressent aux produits agricoles, comme ressources touristiques (achat direct des dattes chez l'agriculteur). Le reste est attiré par le tourisme culturel (kasbah et ksour, gravures etc.) et notamment celui du désert (randonnées chamelières, traversé des dunes par des quads et des véhicules $4 \times 4$, etc.) qui représente $65 \%$ des désirs touristiques. C'est-àdire, que les touristes utilisent souvent les oasis comme points de départ d'excursions vers le désert, et dont la durée moyenne de séjour ne dépasse guère 1,3 nuitée.

En général, l'activité d'agrotourisme est souvent très limitée dans les 4 palmeraies amont de la vallée (Mezguita, Tinzouline, Ternata, Fezouata). Cette activité exercée par une minorité d'agriculteurs se présente, dans les meilleures conditions, sous forme de restauration ou d'hébergement dans l'oasis «chez habitant », les randonnées à dos des mulets. Ce contact permet aux touristes la découverte du monde agricole, de son patrimoine, de ses produits et de son savoir-faire (Photo 1).

A partir de la palmeraie de Ktaoua et notamment à $\mathrm{M}^{\prime}$ hamid et ses lisières dunaires « bivouacs en plein espace dunaire, coucher de soleil sur la crête des dunes» commence la culture du nomadisme et se développe le tourisme du désert. L'hébergement est géré le plus souvent en dehors des acteurs agricoles, et dirigé par des agences de voyage qui sont parfois hors de la zone. 

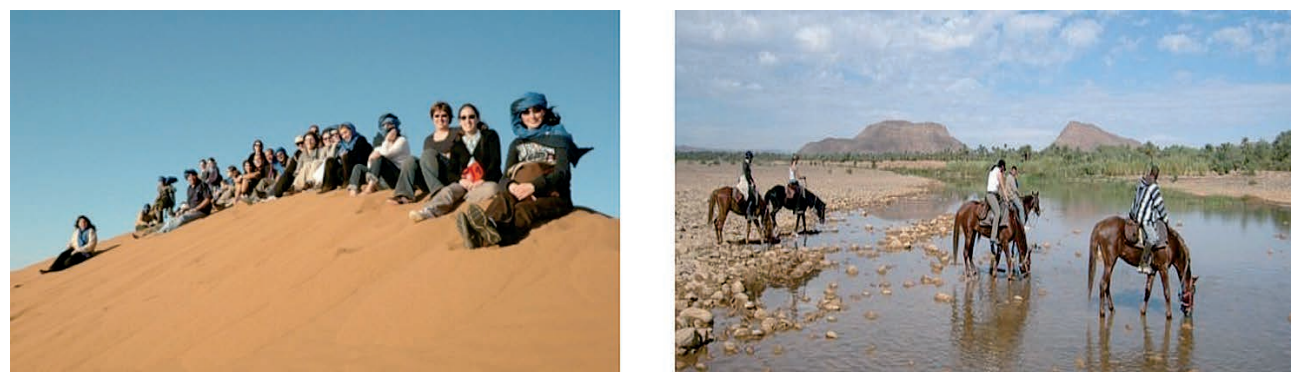

Photo 1 - Coucher de soleil à la dune de Tinfou et randonnées à dos des mulets (source : Aziz Bentaleb, 2016)

Pour prendre l'exemple de la France, légalement l'agritourisme est défini par l'article 2 de la loi du 30.12.88 : neufs activités agrotouristique existent sous la marque Bienvenue à la Ferme (marque déposée par le service agriculture et tourisme de l'APCA) : ferme-auberge, produits de la ferme (avec un point de vente à la ferme), goûter à la ferme, ferme équestre, chasse à la ferme, ferme de découverte, ferme pédagogique (labellisée par le service agriculture et tourisme depuis 1996), ferme de séjour (gîte, couvert et loisirs sur place ou à proximité d'autres prestataires de services ; un label sous réserve de respecter les chartes des chambres et tables d'hôtes de Gîtes de France), camping à la ferme (Disez, 1996 et ENITAC, 1995). Cependant, au Maroc l'agrotourisme n'est pas reconnu sur le plan législatif, même si les oasis constituent un laboratoire de ressources et des savoir-faire agricoles authentiques et ancestraux qui méritent la valorisation par le tourisme. On peut aussi introduire dans la zone des activités d'agrotourisme, supervisées par le Ministère d'agriculture et celui de tourisme sous la marque; bienvenue à la palmeraie, bienvenue à la diversité culturelle et ethnique, accueil oasispalmier ; dune-nomade, goûter le thé à la vallée, goûtez les dattes, dormir au ksar et à la kasbah, randonnées de Jbel Saghro et Bani, se restaurer au bivouac, etc.

\section{L'archi-tourisme et le ciné-tourisme : Pour une meilleure valorisation du produit du terroir}

Le patrimoine architectural dans la zone constitue un véritable musée en plein air, très diversifié et enraciné dans le passé, comme en témoignent la densité et l'originalité de ses aspects matériels le long d'oued Draa. Ces sites patrimoniaux sont un atout majeur qui exprime l'originalité dans l'emploi des matériaux, l'adoption des formes, la fonction, le rôle 
et l'utilité de chaque construction (Boussalh, 2001).

Malheureusement, ces superbes bâtisses se dégradent et sont laissées à l'abandon. Elles tombent en ruine pour des raisons d'ordre climatique, socioéconomique et politique. D'où la nécessité d'élaborer des plans de sauvegarde et de réhabilitation de ce patrimoine, afin de préserver l'identité locale et de promouvoir le développement humain.

Les investigations empiriques montrent que la zone recèle des potentialités favorables pour l'attrait des touristes ambitieux voulant connaitre le monde fermé des ksours et kasbahs et rencontrer les ksouriens. Ceci constitue pour les acteurs locaux une occasion pour transmettre des messages culturels relatifs à l'ingéniosité des maâlmines (bâtisseurs) oasiens. Il s'agit d'expliquer aux visiteurs que cet habitat en terre qui marque l'image du désert, constitue un produit d'histoire et de société d'une architecture traditionnelle, mais cette fois sans architectes. Les techniques de construction utilisées, la morphologie des ruelles pour bénéficier des microclimats locaux, les motifs géométriques, la diversité linguistique de la population et la stratification socio-spatiale des ksour (et kasbah) ont mis en évidence le génie savoir-faire des autochtones (Bentaleb, 2013). Ce mode d'organisation saharien a déjà attiré l'attention des touristes chercheurs en ethnologie, anthropologie, sociologie, en géographie, etc., considérant que la disparation de cette architecture vernaculaire en terre constitue une perte irrécupérable pour le Maroc et pour le patrimoine de l'humanité.

Pour valoriser cet habitat en pisé à l'échelle locale, l'école de l'architecture de Lausanne en collaboration avec le CERKAS ${ }^{2}$, organise à plusieurs reprises des voyages d'étude dans la vallée du Draa. Les étudiants architectes sont accueillis à Assalim dans la maison d'Aït Caid pour étudier les matériaux de pisé et la réhabilitation des constructions en terre ${ }^{3}$. Ce procédé scientifique a mis en valeur l'importance de cet exceptionnel habitat en terre qui pourrait contribuer à renforcer une image touristique de la zone.

2 Centre de Conservation et de Réhabilitation du Patrimoine Architectural des Zones Atlasiques et Subatlasique

3 Entretien avec le prof. Mohamed Ait Hamza, ex-Directeur du CEHE à IRCAM. 
Ainsi, la diversité des paysages, la richesse architecturale la diversité ethnique, la lumière exceptionnelle font du Draa l'un des lieux les plus visités par les cinéastes au Maroc. Parmi les films tournés dans la région: The Last Temptation of Christ, Un thé au Sahara, Marry the mother of jesus, Ancient Egyptians, Maosus, Kundun, Saint Paul, Jesus Family's tree, Crusades, Babel....

Donc, le patrimoine architectural peut être valorisé par la création des circuits touristiques et le développement de l'industrie cinématographie, à travers :

- La protection législative de l'architecture en terre. On remarque en général, la non-reconnaissance de ces constructions et des matériaux (architecture en terre) utilisés par la loi semble constituer le plus grand handicap à leur durabilité. En fait, les architectures en pisé sont toujours considérées vétustes et non résistantes. Souvent sans titre foncier, ces bâtiments ne sont ni acceptés comme fonds, ni comme immeuble par les banques (Ait Hamza, 1992).

- L'encouragement des cinéastes lors des rendez-vous de film transsaharien, (initié par l'association de Zagora) à animer des ateliers autour des métiers du cinéma en faveur des artisans et des acteurs locaux.

- La transformation de l'architecture traditionnelle en écomusées ruraux dans chaque palmeraie, en exposant le savoir-faire culturel millénaire des oasiens. Des exemples existent déjà :(musée des Arts et Traditions de la Vallée du Drâa, musée Oulad Driss : les habitants ont rassemblé les objets quotidiens de la vie traditionnelle dans le Désert. Dans ce Musée, on peut découvrir les habits caractéristiques de chacune des ethnies de la région, les outils du travail de la terre ou des caravanes de dromadaire ainsi que des ustensiles pour la cuisine, et la fabrication du couscous...).

- La transformation des kasbahs en complexes culturels ou en chambres d'hôtes. Des initiatives privées ont déjà permis la transformation de kasbah en hôtels ou en restaurant, sans autant leur faire perdre leur 
cachet d'authenticité dans sa forme globale.

- La classification d'autres sites d'intérêts civilisationels sur la liste du patrimoine national, voire même sur la liste du Patrimoine Mondial de l’Humanité.

- Mener des campagnes de sensibilisation nationale et locale pour la sauvegarde de ce patrimoine rural, avec la participation de la population locale dans tous les projets territoriaux.

- La création de labels et de normes pour les produits touristiques et cinématographiques seront déterminants pour la qualité du tourisme et la réussite de l'expérience touristique des visiteurs. Le tournage des films dans la zone a pour objectif de mettre en valeur le patrimoine bâti culturel historique et préhistorique et les gravures rupestres, comme langage d'échanges culturel. Les festivals jouent également un rôle non négligeable dans la réhabilitation et l'animation des lieux patrimoniaux. La réutilisation des espaces patrimoniaux pour la tenue de spectacles ou d'expositions est une pratique qui a fleuri au sein des festivals ; elle fait revivre des monuments délaissés.

En général, le développement du tourisme culturel visant la valorisation des produits du terroir archéologiques nécessite :

-Elaboration des plans participatifs en concertation avec tous les acteurs de tourisme pour la sauvegarde des gravures rupestres. Cet aménagement vise non seulement la protection et la valorisation des gravures rupestres pour une destination privilégiée d'écotourisme culturel unique du genre, mais aussi l'encouragement de la recherche scientifique par des missions des études empiriques.

- Élargissement du système de gardiennage pour les arts rupestres les plus menacés, et l'installation de panneaux signalétiques à l'entrée de quelques sites majeurs. Les panneaux en question devraient contenir un extrait du texte de la loi 22-80, écrit en tifinagh ( $l^{\prime}$ alphabet amazighe) en arabe, en français et en anglais.

- Mener des campagnes de sensibilisation auprès des autorités locales et la population riveraine de l'aire rupestre, ainsi que l'implication des communes et du tissu associatif dans la gestion et la mise en valeur des sites archéologique, qui constitue une référence culturelle 
et historique collective à tous les oasiens.

- Création des itinéraires culturels regroupant un grand nombre des palmeraies du Draa moyen et de Jbel Bani, organisés autour d'un thème $\mathrm{d}$ 'intérêt historique, artistique et social. Cette thématique a une véritable connotation oasienne de par son contenu, sa signification et son tracé géographique. L'itinéraire est constitué d'un large panel institutionnel; et son implantation intervient dans son fonctionnement avec différents acteurs (administrations publiques, société civile, agents de développement local, musées, centres d'interprétations, archéologues, gestionnaires du Patrimoine local, etc.). La réalisation des chemins de l'art rupestre et culturel ainsi que désertique permettra à générer une offre touristique thématique et rigoureuse qui intègre les notions de nature et culture, de paysage et patrimoine culturel, qui peuvent contribuer au développement durable des communautés locales sur lesquelles se situe L'itinéraire.

Il serait donc serait vivement nécessaire que les autorités concernées se penchent sur la question de valorisation et de classification des biens archéologiques de la zone sur la liste du patrimoine mondial de l'UNESCO.

\section{Conclusion}

Le Draa recèle un gisement touristique riche, rude et cependant très fragile. Le potentiel historique, artistique, artisanal et agritouristique est mal exploité afin de développer un tourisme rural générateur de revenu, et par conséquent le Draa constitue un lieu de passage d'une clientèle importante. L'agrotourisme et le tourisme culturel sont des éléments clés pour sauvegarder l'environnement oasien et faciliter les liens sociaux entre personnes appartenant à des catégories sociales et à des territoires différents. Ceci peut être perçu comme une alternative permettant de rencontrer et de vivre avec les autochtones dans une relation d'échange culturel. C'est donc un créneau à promouvoir dans la zone. A ce titre, le développement local doit mettre l'accent sur l'initiative et le savoir-faire local et faire appel à la dimension culturelle comme étant le vecteur de développement le plus assuré comme composante pour donner un élan à l'économie locale.

La mise en valeur touristique des produits du terroir est devenue 
un outil efficace de valorisation des ressources patrimoniales, de développement et de lutte contre la pauvreté et le désenclavement de cette région marginalisée, en contribuant à son «redéveloppement» tout en créant des espaces porteurs de petits projets rentables et durables. Le tourisme devrait avoir un rôle important dans le développement des cultures locales et des arts populaires à travers l'installation de musées ethnologiques et culturels. Ce qui permet de garder une part de l'histoire locale et de préserver certains monuments culturels, historiques et archéologiques et certaines traditions. Les exemples de sauvegarde de sites pareils par le tourisme sont innombrables. Réconcilier tourisme et culture serait possible c'est le comment qui reste à définir.

\section{Références bibliographiques}

Ait Hamza M. (1992), L'habitat dans le Dadès et le rôle de l'émigration dans son évolution récente, In : la recherche scientifique au service du développement. Pub. FLSH, Rabat, Série Colloque et Séminaires N²2, pp : 127-146.

Bentaleb, A (2015), dynamique de désertification dans les oasis du Draa moyen: Analyses et perspectives ». Edition, Université Européenne, Saarbrucken, Deutschland/ Allemagne. ISBN:978-3-8416-6443-3. 340p.

Bentaleb, A (2013) «Impact du tourisme présaharien sur les ressources patrimoniales dans la vallée du Draâ moyen, cas de la palmeraie de M'hamid. In : Ressources patrimoniales et alternatives touristiques entre oasis et montagnes. Coordination : Mélanie Duval, Véronique Peyrache-Gadeau et Mohamed Oudada. Collection Edytem $N^{\circ}$ 14, juin 2013. Pp 25-34. Publication Edytem, Université de Savoie et du CNRSP.

Boussalh, Mohammed (2001) «L'habitat vernaculaire en terre des vallées présahariennes du Maroc : cas des vallées du Drâa, in : Le patrimoine culturel africain, Publication de l'Université Senghor, Ed. Maisonneuve/ Larose, Paris, 2001 (pp.213-225).

Diser, N. (1996), Agritourisme et développement territorial. Exemples du Massif Central ; Thèse de Doctorat. Université Blaise Pascal de Clermont Ferrand. 240p.

ENITAC, (1995), Agritourisme et développement local, Ed. ENITA ClermontFerrand. Collection « Actes » $N^{\circ}$ 3. Juin 1995. $140 \mathrm{p}$. 
Jacques, P et Emmanuelle, M, (2001), L'agritourisme en 2001, la diversité des territoires et des acteurs: entre témoignage et commerce, Direction de l'espace rural et de la forêt, Pp 56-78. 